\title{
Intraventricular Neurocysticercosis and Bruns' Syndrome: A Review
}

Breanna R Campbell, David Reynoso, and A Clinton White Jr.*

Infectious Disease Division, Department of Internal Medicine, University of Texas Medical Branch, Galveston, TX

Article Info

\section{Article Notes}

Received: December 21, 2016

Accepted: February 07, 2017

\section{${ }^{*}$ Correspondence:}

A Clinton White, MD

Infectious Disease Division

Department of Internal Medicine

301 University Boulevard

Galveston, TX 77555-0435, E-mail: acwhite@utmb.edu

(c) 2017 A Clinton White. This article is distributed under the terms of the Creative Commons Attribution 4.0 International License.

\section{Keywords}

Neurocysticercosis

Taenia solium

Obstructive hydrocephalus

Neuroendoscopy

\section{ABSTRACT}

Neurocysticercosis, caused by Taenia solium, is a common cause of neurologic disease worldwide. Approximately 2,000 cases per year are diagnosed in the United States. Intraventricular neurocysticercosis is a severe form of the disease, in which cysticerci within the cerebral ventricles cause obstructive hydrocephalus. Symptoms of hydrocephalus include headache, nausea, vomiting, altered mental status, dizziness, and decreased visual acuity. In some cases, sudden onset of symptoms are associated with changes in head position leading to acute obstruction (Bruns' syndrome). Diagnosis depends on neuroimaging studies, especially 3 dimensional MRI sequences. Optimal treatment involves relief of hydrocephalus by removal of the cysticerci. For cysticerci in the lateral and third ventricles, this can usually be accomplished via minimally invasive surgery (neuroendoscopy). However, this is not possible with adherent cysticerci. In some cases of cysticerci in the $4^{\text {th }}$ ventricle, open surgical microdissection via an occipital approach may be safer. Mortality is rare with appropriate management.

\section{Introduction}

Cysticercosis refers to infection by the larval stage of the pork tapeworm Taenia solium, whose clinical manifestations include neurocysticercosis (NCC) and extraneural cysticercosis. NCC is divided into parenchymal and extraparenchymal forms; the latter includes intraventricular, subarachnoid, and occasionally spinal. Intraventricular NCC (IVNCC) can be rapidly progressive and fatal if untreated. There is an emerging consensus in the literature for treatment of IVNCC. Here we review the epidemiology, presentation, diagnosis, and current management recommendations of IVNCC.

NCC is the most common helminthic neurological infection in the world, and one of the most frequent causes of adult-onset epilepsy and hydrocephalus worldwide ${ }^{1}$ Globally, NCC is reported to affect approximately 50 million individuals, mainly in Latin America, subSaharan Africa, and South and Southeast Asia ${ }^{2}$. A meta-analysis noted that $29 \%$ of people with epilepsy in areas endemic to T. solium have NCC ${ }^{3}$. Given the limited availability of advanced diagnostic imaging modalities in endemic areas, infection prevalence is likely underestimated ${ }^{2}$.

Based on reviews of a national sample of discharge summaries for the years 2003-2015, there were estimated to be over 18000 U.S. hospitalizations for NCC, leading to a healthcare cost greater than USD $\$ 900$ million $^{4}$, with $1320-5050$ new cases diagnosed per year ${ }^{5}$.

\section{The Disease}

\section{Parasite life cycle}

T. solium infects pigs as intermediate hosts, with humans serving 
as both intermediate and definitive hosts. When a person eats undercooked pork meat containing a viable cysticercus, the larval stage of T. solium, the scolex evaginates, and the suckers and hooks of the scolex attach to the intestinal mucosa. The ensuing maturation and intestinal infestation by adult tapeworms is known as taeniasis, and is characterized by largely-asymptomatic shedding of large numbers of proglottids and eggs in stool ${ }^{6}$. Pigs acquire cysticercosis by ingesting eggs or proglottids shed by the tapeworm carrier. Humans also acquire cysticercosis by ingesting the T. solium eggs via autoinfection or from another human carrier of tapeworm eggs. After ingestion, the eggs hatch and release the invasive larvae (termed oncospheres); these invade the intestinal wall and spread through the bloodstream. While cysticerci may develop in a wide range of organs, most symptomatic disease results from infection of the central nervous system, subcutaneous tissue, and eyes ${ }^{6}$.

\section{Extraparenchymal NCC}

Most patients with neurocysticercosis present with cystic lesions or calcifications in the brain parenchyma. Seizures are the most common presenting symptom of parenchymal $\mathrm{NCC}^{1}$. In contrast, the main presentation of IVNCC or subarachnoid NCC is with symptoms of raised intracranial pressure such as headache, nausea, vomiting, or dizziness ${ }^{6}$. Extraparenchymal NCC refers to involvement of the ventricles, subarachnoid space, and occasionally the spine; mixed forms are common. Subarachnoid disease often involves the basilar cisterns and Sylvian fissures. In some cases, the parasites transform into proliferating membranes termed racemose cysticercosis. In spinal NCC, the leptomeningeal spaces are frequently involved, and cysts may inhabit the spinal cord itself. Spinal involvement was previously considered rare, but appears to be seen frequently in patients with basal subarachnoid $\mathrm{NCC}^{7}$. Subarachnoid NCC may present with a range of clinical manifestations including communicating hydrocephalus, mass lesions, stroke, and meningismus ${ }^{1}$.

IVNCC is the presenting form of NCC in approximately 10 $20 \%$ of patients worldwide ${ }^{6}$. Most often, extraparenchymal NCC presents with symptoms of hydrocephalus, carrying a high mortality rate ${ }^{1}$. In the U.S., approximately $16 \%$ of NCC cases present with hydrocephalus ${ }^{8}$. A review of inpatient cysticercosis deaths from 1998 to 2011 found obstructive hydrocephalus in $27 \%$ of deaths ${ }^{9}$. When viable, the cysts are often freely mobile and may lodge in foramina or the aqueduct. The resulting mechanical obstruction is the most frequent cause of hydrocephalus, whether occurring at the ventricles; the aqueduct of Sylvius; or the foramina of Monro, Luschka, or Magendie.

Hydrocephalus typically presents with headache, nausea, vomiting, altered mental status, and decreased visual acuity associated with papilledema ${ }^{6}$. Acute hydrocephalus can lead to sudden death by brainstem herniation, displacement, or distortion ${ }^{10}$; or may present strikingly in the form of Bruns' syndrome, with sudden attacks of severe headache, nausea/vomiting, and vertigo, brought on by abrupt head movement ${ }^{11}$. This constellation of symptoms is due to episodic hydrocephalus related to intermitted obstruction of cerebrospinal fluid (CSF) flow by ball-valve movement of intraventricular cysts. Bruns syndrome was originally described in fourth ventricular NCC, but can also be caused by third ventricular cysts, tumors ${ }^{12}$, or even tuberculomas ${ }^{13}$.

\section{Diagnosis}

Diagnosis of NCC can be difficult; in IVNCC, diagnosis is usually straightforward and based primarily on imaging studies with confirmation from serologic tests.

\section{Serology}

A number of serologic tests are available for the diagnosis of neurocysticercosis. Most tests employing crude antigens (including all commercially available ELISAs) are associated with poor sensitivity and specificity. The only reliable test for antibody in cysticercosis is the enzymelinked immunotransfer blot (EITB) assay. While the sensitivity is not optimal for single parenchymal cysticeri or for calcified disease, the sensitivity and specificity of EITB are close to $100 \%$ for IVNCC ${ }^{14}$. EITB carries a greater sensitivity on serum samples as compared to CSF samples (100\% to $85.7-90.2 \%$, respectively) ${ }^{15}$. Therefore, there is essentially no advantage to using CSF for EITB assays.

\section{Imaging}

Non-contrast CT scanning is sensitive for calcified and parenchymal lesions, but is insensitive for extraparenchymal disease ${ }^{15}$. Thus, MRI is the preferred diagnostic test for extraparenchymal NCC (Figure 1). On either CT or MRI, viable cysticerci are small and round, with thin walls and fluid isodense to CSF. T1 sequences may reveal a dense nodule within the cyst ("hole-in-dot"). Given the complementary nature of CT and MRI with regard to NCC, both modalities are recommended for diagnosis. A study on the role of conventional MR imaging sequences in the evaluation of NCC found that a T1-gadolinium series acquired 8 minutes after contrast injection was more specific for total NCC lesions than fluid attenuation inversion recovery (FLAIR), T1 and T2 series, and early T1-gadolinium images ${ }^{16}$. Gadolinium-enhanced MRI (Gd-MRI) may aid in detecting ependymitis ${ }^{17}$, a relative contraindication to neuroendoscopy ${ }^{18}$. However, Gd-MRI is not proven to characterize subarachnoid or intraventricular NCC, as these cysts have similar signal intensity to $\mathrm{CSF}^{16}$.

Newer MRI acquisition techniques promise improved detection of IVNCC, including fast imaging employing 


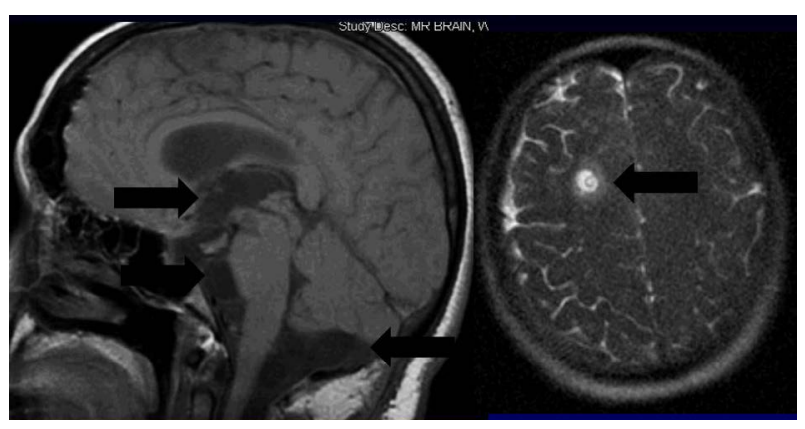

Figure 1: Sagittal view T1 (a) and T2 (b) images from a patient with neurocysticercosis involving the (a.) 3rd ventricle, basilar cisterns, and (b.) parenchyma.

steady-state acquisition (FIESTA), three-dimensional constructive interference in steady state (3D CISS; a modified FIESTA sequence), T2 star-weighted angiography (SWAN), and spoiled gradient recalled echo sequence (SPGR). Thus far, FIESTA and SPGR have demonstrated the best sensitivity in detecting IVNCC ${ }^{19}$. FIESTA is more sensitive than SPGR in demonstrating the cysticercus membrane in subarachnoid NCC, while FLAIR is more sensitive than T2 (but not T1) SPGR ${ }^{20}$. Similarly, SWAN has demonstrated improved detection of IVNCC as compared to traditional $\mathrm{MRI}^{21}$. Further, 3D CISS has been used to diagnose previously missed obstructive membranes in IVNCC ${ }^{22}$.

\section{Management}

\section{Emergent stabilization}

Treatment of neurocysticercosis includes managing hydrocephalus, seizures, and the infection itself. Initial attention to life threatening symptoms is imperative, most notably increased intracranial pressure (ICP). While there may be benefit to anti-inflammatory therapy such as steroids in some cases, neurosurgical intervention is key in patients with increased ICP ${ }^{1}$. Antiparasitic therapy designed to eliminate the infection is never an emergency, and is contraindicated in the setting of increased ICP ${ }^{15}$.

\section{Neuroendoscopy and minimally invasive approaches}

The traditional approach for increased ICP related to IVNCC is placement of an external ventricular drain (EVD) or ventriculoperitoneal shunt (VPS) to relieve pressure. However, shunt failure is common, and mortality in these cases remains high ${ }^{1}$. Furthermore, relief of hydrocephalus via EVD or VPS may make definitive therapy by neuroendoscopy more difficult. Antihelminthics may decrease the rate of shunt failure, but should not be given if cyst removal is planned ${ }^{15}$.When possible, cyst removal is the mainstay of management of IVNCC with obstructive hydrocephalus. Neuroendoscopy is now considered the primary procedure for relief of IVNCC-related hydrocephalus. Under direct endoscopic visualization, cystercerci appear as a "full moon:" this endoscopic sign is considered pathognomonic for IVNCC. Even in the emergent case of Bruns' syndrome, neuroendoscopy has been proven both diagnostic and curative $^{23}$, though it occasionally requires endoscopic third ventriculostomy (ETV) or septum pellucidotomy to treat hydrocephalus ${ }^{24,25}$. These approaches require a practiced hand and are not without their risks, which include intraventricular bleeding ${ }^{26}$, memory loss, hemiparesis, mutism, and aphasia ${ }^{24}$. It should be noted that multiple studies wherein cysts have ruptured during endoscopy have not been complicated by subsequent development of ventriculitis or arachnoiditis ${ }^{27}$. Finally, antiparasitic therapy after IVNCC cyst excision may not be necessary. A systematic review found that patients who underwent surgical removal of a single intraventricular cyst and received antiparasitic therapy afterward had a lower rate of delayed hydrocephalus than those who did not ${ }^{27}$, but a large case series from the NIH has not confirmed this finding ${ }^{28}$.

\section{Fourth ventricle neuroendoscopy and open surgery}

While the fourth ventricle is a common site of IVNCC, reports of successful flexible neuroendoscopy for fourth ventricle extirpation are limited compared to that of lateral or third ventricles. Transventricular, transforaminal flexible endoscopy and transventricular, transaqueductal scope-inscope endoscopy have proven effective in extirpation of fourth ventricle cysts ${ }^{10,18,29}$. Endoscopy was performed in conjunction with ETV in these cases, and shunt placement was avoided in each patient. However, both involve passage of the scope through the aqueduct, which poses risks for major neurologic damage. Open surgical microdissection for fourth ventricular NCC requires suboccipital craniotomy, but is generally well tolerated. However, compared to endoscopic approaches, open surgery may lead to increased operative time, blood loss, and hospitalization ${ }^{24}$, and delayed hydrocephalus commonly occurs ${ }^{30}$.

\section{Adherent cysticerci}

Cyst death releases antigenic material into the ventricles, leading to ventriculitis ${ }^{31}$. Localized reactions may then fixate the cyst capsules to the walls of the ventricles or to the subarachnoid tissue, causing adherent cysticerci (Figure 2). These, in turn, may also block CSF flow and predispose the patient to sustained hydrocephalus and elevated ICP. In such cases, surgical involvement is usually not possible without causing damage to nearby structures $^{32}$. Instead, patients should be managed by CSF diversion without cyst extirpation ${ }^{18,32}$, although partial resection via flexible neuroendoscopy with additional ETV has proven successful ${ }^{29}$.

\section{Summary}

Neurocysticercosis presents with a pleomorphic clinical picture including potentially fatal intraventricular 


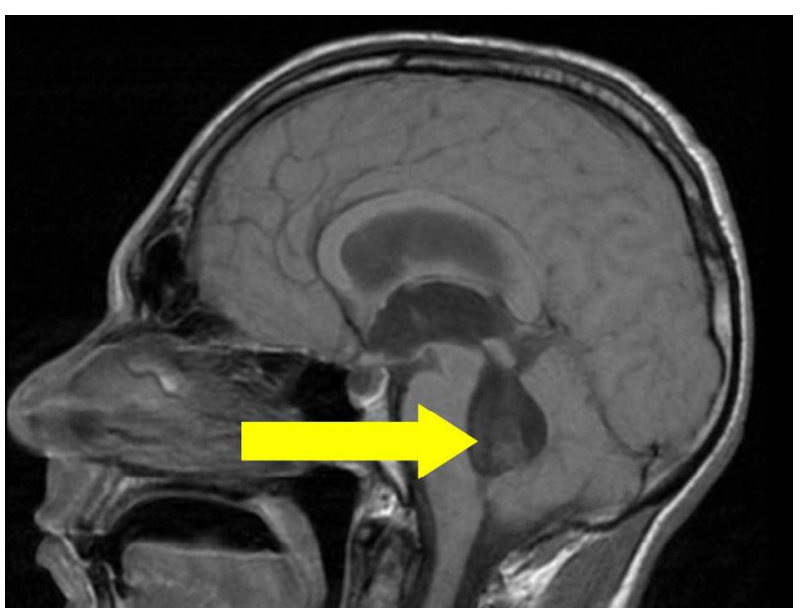

Figure 2: T1 MRI demonstrating a degenerating cyst adherent to the wall of the 4 th ventricle.

neurocysticercosis, which usually presents with symptoms of obstructive hydrocephalus. Advanced MRI imaging modalities improve the sensitivity of diagnosis of IVNCC and aid in guiding intervention. Flexible neuroendoscopy has emerged as the surgical approach of choice when possible. Adoption of neuroendoscopy as an initial and primary intervention for treatment of IVNCC is likely to improve outcomes for patients suffering from this disease. However, many neurosurgeons prefer open microdissection for cysticerci in the fourth ventricle. Adherent cysticerci often cannot be removed; patients instead require shunting for relief of obstructive hydrocephalus, followed by steroids and antiparasitic drugs to lessen the risk of shunt failure.

\section{References}

1. García HH, Nash TE, Del Brutto OS. Clinical symptoms diagnosis and treatment of neurocysticercosis. Lancet Neurol. 2014; 13: 1202-1215.

2. World Health Organization. Working to overcome the global impact of Neglected Tropical Diseases. First Report on Neglected Tropical Diseases. Geneva, Switzerland: World Health Organization 2010. Available: http://www.who.int/neglected_diseases/2010report/en/. Accessed 29 October 2016.

3. Ndimubanzi PC, Carabin H, Budke CM, et al. A Systematic Review of the Frequency of Neurocyticercosis with a Focus on People with Epilepsy. PLoS Negl Trop Dis. 2010; 4(11): e870.

4. O'Neal SE, Flecker RH. Hospitalization frequency and charges for neurocysticercosis United States 2003-2012. Emerg Infect Dis. 2015 June; 21(6): 969-976.

5. Serpa JA and White AC. Neurocysticercosis in the United States. Pathog Glob Health. 2012 Sep; 106(5): 256-260.

6. Garcia HH, Coyle CM, White AC. Cysticercosis Tropical Infectios Diseases Principles Pathogens and Practice $3^{\text {rd }}$ Edition. ElsevierSaunders Philadephia. 2011; 815-823.

7. Callacondo D, García HH, Gonzales I, et al. Cysticercosis Working Group in Peru High frequency of spinal involvement in patients with basal subarachnoid neurocysticercosis. Neurol. 2012 May; 78: 13921400 .

8. Wallin MT, Kurtke JF. Neurocysticercosis in the United States: review of an important emerging infection. Neurol. 2004; 63(9): 1559-1564.
9. O’Keefe KA, Eberhard ML, Shafir SC, et al. Cysticercosis-Related Hospitalizations in the United States 1998-2011. Am J Trop Med Hyg. 2015 Feb 4; 92(2): 354-359.

10. Goel RK, Ahmad FU, Vellimana AK, et al. Endoscopic management of intraventicular neurocysticercosis. J Clin Neurosci. 2008 Oct; 15(10): 1096-1101.

11. Bruns L. Neuropathologische Demonstrationen. Neurol Zentralbl. 1902;21:561-567.

12. Krasianski M, Müller T, Stock K, et al. Bruns Syndrome Caused by Intraventricular Tumor. Eur J Med Res. 2008; 13: 179-181.

13. Lu Z, Zhang B, Qiu W, et al. Disseminated Intracranial Tuberculoma Mimicking Neurocysticercosis. Intern Med. 2011; 50: 2031-2034.

14. Rodriguez S, Dorny P, Tsang VSW, et al. Detection of Taenia solium Antigens and Anti-T solium Antibodies in Paired Serum and Cerebrospinal Fluid Samples from Patients with Intraparenchymal or Extraparenchymal Neurocysticercosis. J Infect Dis. 2009; 199: 13451352.

15. Webb C, White AC. Update on the Diagnosis and Management of Neurocysticercosis. Curr Infec Dis Rep. 2016 Dec; 18: 44.

16. Lucato LT, Guedes MS, Sato JR, et al. The Role of Conventional MR Imaging Sequences in the Evaluation of Neurocysticercosis: Impact on Characterization of the Scolex and Lesion Burden. Am J Neuroradiol. 2007 Sep; 28: 1501-1504.

17. Zee CS, Segall HD, Destian S, et al. MRI of intraventricular cysticercosis: surgical implications. J Comput Assist Tomogr. 1993 Nov-Dec; 17(6): 932-939.

18. Sinha S, Sharma BS. Intraventricular neurocysticercosis: a review of current status and management issues. Br J Neurosurg. 2012 Jun; 26(3): 305-309.

19. Mont'Alverne Filho FE, Machado LR, Lucato LT, et al. The role of 3D volumetric MR sequences in diagnosing intraventricular neurocysticercosis: preliminary results. Arg Neuro-Psiquiatr. 2001 Feb; 69(1): 74-78.

20. Carrillo Mezo R, Lara García J, Arroyo M, et al. Relevance of 3D magnetic resonance imaging sequences in diagnosing basal subarachnoid neurocysticercosis. Acta Trop. 2015; 152: 60-65.

21. Neyaz Z, Patwari SS, Paliwal VK. Role of FIESTA and SWAN sequences in diagnosis of intraventricular neurocysticercosis. Neurol India. 2012; 60: 646-647.

22. Dinçer A, KohanS, Ozek MM. Is all Communicating hydrocephalus really communicating Prospective study on the value of 3D-Constructive interference in steady state sequence at 3T. AJNR Am J Neuroradiol. 2009; 30: 1898-1906.

23. Torres Corzo J, Rodriguez Della Vecchia R, Rangel-Castilla L. Bruns syndrome caused by intraventricular neurocysticercosis treated using flexible endoscopy. J Neurosurg. 2006; 104: 746-748.

24. Bergsneider M, Holly LT, Lee JH, et al. Endoscopic management of cysticercal cysts within the lateral and third ventricles. J Neurosurg. 2000; 92: 14-23.

25. Teegala R, Rajesh KG, Raviprasad VY, et al. Emergency Neuroendoscopic Management of Third Ventricular Neurocysticercosis Cyst Presented with Bruns Syndrome: Report of Two Cases and Review of Literature. J Korean Neurosurg Soc. 2014; 55(3): 173-177.

26. Psarros TG, Krumerman J, Coimbra C. Endoscopic management of supratentorial ventricular neurocysticercosis: case series and review of the literature. Minim Invasive Neurosurg. 2003 Dec; 46(6): 331334.

27. Khade P, Lemoa RS, Toussaint LG. What is the Utility of Postoperative Antihelminthic Therapy After Resection for Intraventricular Neurocysticercosis. World Neurosurg. 2013; 80(5): e141-e142. 
28. Nash TE, Ware JM, Mahanty S. Ventricular neurocysticercosis in the United States: treatment complications and outcome in a tertiary referral center (\#34). American Society of Tropical Medicine and Hygiene (ASTMH): 65th Annual Meeting. Session 10 - Cestodes: Cysticercosis and Echinococcosis. November 14, 2016. Atlanta, GA, USA

29. Husain M, Jha DK, Rastogi M, et al. Neuro endoscopic management of intraventricular neurocysticercosis (NCC). Acta Neurochirurgica. 2007 Apr; 149(4): 341-346.
30. Rangel-Castilla L, Serpa JA, Gopinath SP, et al. Contemporary neurosurgical approaches to neurocysticercosis. Am J Trop Med Hyg. 2009 Mar;80(3):373-8.

31. Cuetter AC, Garcia Bobadilla J, Guerra LG, et al. Neurocysticercosis: Focus on Intraventricular Disease. Clin Infect Dis. 1997; 24: 157-164.

32. Cuetter AC, Andrews RJ. Intraventricular Neurocysticercosis: 18 Consecutive Patients and Review of the Literature. Neurosurg Focus. 2002; 12(6): e5. 